\title{
Fluid exchange under scleral contact lenses in relation to wearing time
}

\author{
L. KO, D. MAURIGE, AND M. RUBEN \\ From the Institute of Ophthalmology and Moorfields Eye Hospital, London
}

Scleral contact lenses are extensively prescribed in certain centres, especially when the $\frac{\vec{\omega}}{\partial}$ eyes to be fitted are abnormal. The original sealed lens prevented the tear fluid behind it from exchanging with the lacrimal secretion present in the conjunctival sac. In con- $f$ sequence, the corneal epithelium rapidly became depleted of oxygen, and this resulted after a few hours in the misting of vision and the appearance of haloes (Smelser, 1952). Oo

In order to promote the circulation of the fluid over the cornea, scleral lenses have been 을 designed with fenestrations or with channels. There appear, however, to have been no $\vec{c}$ objective tests of the effectiveness of these procedures. One way to ascertain this is to stain the fluid behind the lens with fluorescein and to measure the rate at which the $\overrightarrow{0}$ fluorescence is lost; this rate of loss should represent the rate at which unstained tear secretion replaces the original fluorescein-stained fluid. Fluorescein was used for this purpose in the pioneer experiments of Smelser (1952), but a specially designed lens had to be made to collect the dye for measurement at the end of a test, and repeated tests were required to determine the rate of loss.

The availability of the slit-lamp fluorophotometer designed by one of us (Maurice, $\stackrel{\mathbb{D}}{\varrho}$ 1963) has made it possible to follow accurately the changes in dye concentration behind a을 normal lens during a single wearing period.

\section{Method}

Patients who had been routinely fitted with scleral lenses at the Contact Lens and Prosthetics? Department of Moorfields Eye Hospital, High Holborn, were used as subjects for these tests. Three $\frac{}{3}$ patients (four eyes) wore fenestrated lenses (F) and eight patients (ten eyes) channelled lenses $(\mathrm{G})$. The channels were grooves in the haptic from the zones of corneal clearance to the periphery of the lens. The fenestration was $\mathrm{I}$ to $\mathrm{I} \cdot 5 \mathrm{~mm}$. in the limbal clearance zone and was usually sited temporally. The maximum continuous wearing time without misting of vision was noted for each patient. The nature of the test was explained and the lens inserted on to the eye to be tested. Physiological saline buffered to $\mathrm{pH} 7 \cdot 2$ and warmed to body temperature was used as insertion

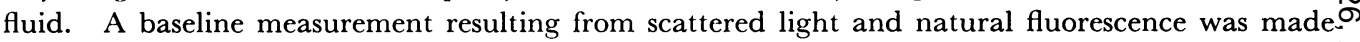
at a point of contact lens clearance from the cornea. The patient then removed the contact lens, $N$ filled it with a solution of ${ }_{10}^{-6} \mathrm{~g} . / \mathrm{ml}$. fluorescein in physiological saline $(\mathrm{pH} 7 \cdot 2)$, and then re- $\underset{\omega}{\mathrm{N}}$ inserted it.

The patient had previously become accustomed to being observed through the fluorophotometer. The clearance zone behind the lens where measurements could be conveniently carried out was identified as quickly as possible, and readings were made at suitable intervals for a period of an hour ${ }^{+}$ or more. Between measurements the patient was allowed normal freedom of movement. At the $\frac{0}{0}$ end of the test the lens was removed, filled with an unstained saline solution, and re-inserted. This $\mathbb{\mathbb { D }}$ allowed a baseline reading to be taken from the same point under the contact lens.

Since most of the lenses were closely fitted apically, the smallest sensitive window of the fluorophotometer (approximately $0.075 \mathrm{~mm}$. wide) had to be employed, and a search had frequently to 
be made to find a region where the lens was sufficiently separated from the corneal surface for the sensitive area to lie entirely within the image of the illuminated slit of fluorescent liquid. Once this was found, the patient could be encouraged to direct his gaze in the appropriate direction during measurements and with experience little difficulty was encountered in obtaining consistent readings.

No unequal mixing of the fluorescent fluid behind the lens, or staining of the cornea, was macroscopically observable in these tests.

\section{Results}

The readings, corrected by the subtraction of the baseline value, were plotted against time on semilogarithmic paper. As would be expected, the points tended to fall on a straight line, and from this the decay of the fluorescence could readily be derived to a sufficient degree of accuracy. The decay was expressed by an exponential coefficient which corresponded to the proportion of the fluid behind the lens which was replaced in a given time interval.

This coefficient varied enormously between different subjects. Fig. I illustrates the results from $F_{I}$, the patient with the highest values, whose left lens had an exchange coefficient of about 12 per hour, indicating that the fluid behind it was replaced every 5 minutes.

By contrast, Fig. 2 shows the results in $\mathrm{C}_{7}$, a patient in whom the coefficient was only about 0.3 per hour, so that only 30 per cent. of the volume of fluid was exchanged in a

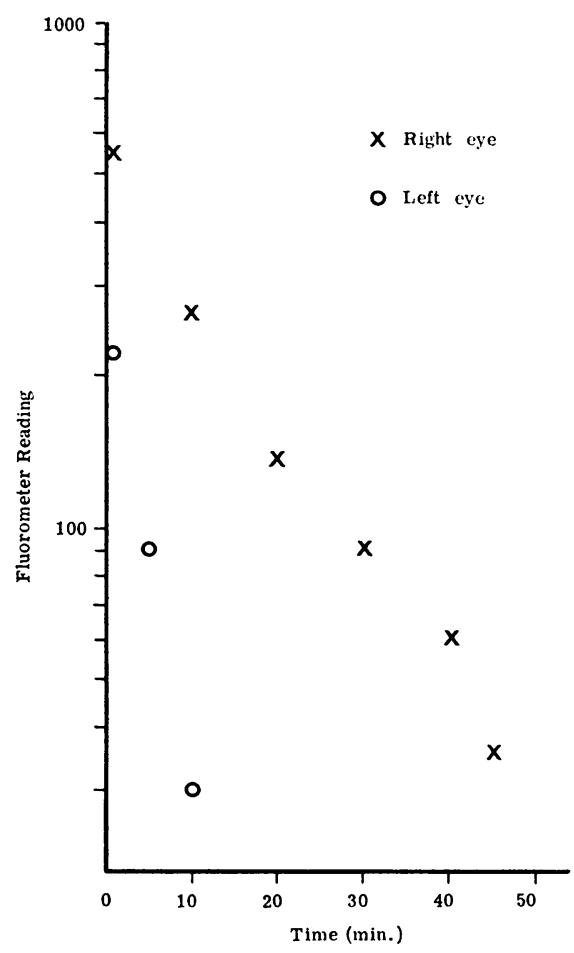

FIG. I Decline in fuorescence behind lenses of patient $F_{\mathrm{I}}$ (fenestrated lenses)

Ordinate: Fluorometer reading (arbitrary units)

Abscissa: Time after insertion of lens 
one-hour period. Two extreme cases demonstrated no appreciable decline in fluorescence over a period of more than one hour, indicating that the lenses were effectively sealed although the channels were functional by clinical tests.

The results obtained from all eleven patients are summarized in the Table where the exchange coefficients are compared with the reported wearing times.

Table Fluorescein exchange rates and wearing times for patients with fenestrated $(\mathbf{F})$ or channelled (C) lenses

\begin{tabular}{|c|c|c|c|c|}
\hline \multirow{2}{*}{$\begin{array}{l}\text { Lenses } \\
\text { Fenestrated }\end{array}$} & \multicolumn{2}{|c|}{ Patient no. } & \multirow{2}{*}{$\begin{array}{c}\begin{array}{c}\text { Exchange } \\
\text { coefficient } \\
\text { (1 hr) }\end{array} \\
\begin{array}{c}3.5 \\
12.0\end{array}\end{array}$} & \multirow{2}{*}{$\begin{array}{l}\begin{array}{l}\text { Wearing } \\
\text { time } \\
(\text { hrs })\end{array} \\
16 \\
16\end{array}$} \\
\hline & $\mathbf{F}_{\mathbf{I}}$ & $\begin{array}{l}\text { RE } \\
\text { LE }\end{array}$ & & \\
\hline & $F_{2}$ & & $\mathrm{I} \cdot 2$ & 4 \\
\hline & $\mathbf{F}_{3}$ & & $2 \cdot 3$ & 4 \\
\hline \multirow[t]{8}{*}{ Channelled } & $\mathrm{Cr}_{\mathbf{r}}$ & & $\mathrm{I} \cdot 8$ & 5 \\
\hline & $\mathrm{C}_{2}$ & & $I \cdot 4$ & 5 \\
\hline & $\mathrm{C}_{3}$ & & 0.5 & 10 \\
\hline & $\mathrm{C}_{4}$ & $\begin{array}{l}\text { RE } \\
\text { LE }\end{array}$ & $\begin{array}{l}0.7 \\
1.6\end{array}$ & $\begin{array}{l}5 \\
4\end{array}$ \\
\hline & $\mathrm{C}_{5}$ & & $2 \cdot 3$ & 2 \\
\hline & C6 & & $3 \cdot 4$ & 4 \\
\hline & $\mathrm{C}_{7}$ & $\begin{array}{l}\text { RE } \\
\text { LE }\end{array}$ & $\begin{array}{l}0 \cdot 2 \\
3 \cdot 4\end{array}$ & $\begin{array}{l}3-4 \\
3-4\end{array}$ \\
\hline & C8 & & $0 \cdot 2$ & $3-4$ \\
\hline
\end{tabular}

\section{Discussion}

The oxygen uptake from the air over the total surface of the human cornea has been measured to be about $6 \mu \mathrm{l}$./hr under normal conditions (Hill and Fatt, ig64). There are no direct experiments to determine what is the smallest uptake required to save the cornea from metabolic embarrassment. The calculations of Fatt, Bieber, and Pye (1969), though beset with assumptions, would suggest that the uptake could drop to $4 \mu \mathrm{l}$. $/ \mathrm{hr}$, $4=\frac{2}{3}$ of the 6 given above before the oxygen consumption of the tissue was restricted, and that at no point would it be entirely suffocated, even if the uptake were as little as $0.6 \mu \mathrm{l} . / \mathrm{hr}$, ten times less than 6.

If the cornea is closed off by a lens, the gas can be brought to its surface only when dissolved in the tear fluid which exchanges with the trapped volume of fluid. Given the solubility of oxygen $\left(0.5\right.$ vol. $\mathrm{O}_{2}$ in 100 vol. $\mathrm{H}_{2} \mathrm{O}$ at $37^{\circ} \mathrm{C}$.), it seems probable that somewhere in the region of $100-1,000 \mu \mathrm{l}$./hr of fresh tear fluid should replace that under the lens in order to satisfy the minimal respiratory demands of the cornea.

It should be emphasized that the exchange coefficients displayed in the Table show only what proportion of the fluid behind the lens is exchanged in an hour. To estimate 
the rate of fluid exchange the actual trapped volume must be known. Thus if we assume the overall clearance to be $0.5 \mathrm{~mm}$., the trapped volume will be about $70 \mu \mathrm{l}$. Then the fastest exchange rate (Fig. I) will correspond to a tear movement under the lens of 840 $\mu \mathrm{l} . / \mathrm{hr}$ and the slow rate (Fig. 2) to one of $20 \mu \mathrm{l} . / \mathrm{hr}$. Put another way, an exchange coefficient of I 3 per hr should correspond to complete satisfaction of the corneal requirements and one of $1 \cdot 3$ per $\mathrm{hr}$ to effective suffocation. Intermediate values might lead to the appearance of visual disturbances after a delay.

Although the actual trapped volumes are uncertain in individual cases, the results shown in the Table are generally explicable from this point of view. Apart from patient $F_{\text {I }}$ only one other $\left(\mathrm{C}_{3}\right)$ had a wearing time greater than $5 \mathrm{hrs}$. These wearing times do not indicate the limit of tolerance but the onset of misting of vision and superficial corneal oedema. Experienced wearers are known to achieve times as long as this even with totally sealed lenses. The conclusion may be drawn, therefore, that the fenestration was totally achieving its purpose only in patient $F_{I}$. Channelling was probably helping none of these patients in respect of oxygen replacement.

\section{References}

FATt, I., BIEBER, M. T., and PYE, S. D. (1969) Amer. F. Optom., 46, 3

HILL, R. M., and FATT, I. (1964) Ibid., 41, 382

MAURICE, D. M. (1963) Exp. Eye Res., 2, 33

SMelser, G. K. (1952) A.M.A. Arch. Ophthal., 47, 328 